\title{
It Works Both Ways: Transfer Difficulties between Manipulatives and Written Subtraction Solutions
}

\author{
David H. Uttal, ${ }^{1}$ Meredith Amaya, ${ }^{1}$ Maria del Rosario Maita, ${ }^{1}$ Linda Liu Hand, ${ }^{1,2}$ \\ Cheryl A. Cohen, ${ }^{1}$ Katherine O'Doherty, ${ }^{3}$ and Judy S. DeLoache ${ }^{4}$
}

${ }^{1}$ Northwestern University, Evanston, Illinois, IL 60208, USA

${ }^{2}$ University of Iowa, Iowa City, Iowa, IA 52242, USA

${ }^{3}$ Vanderbilt University, Nashville, Tennessee, TN 37240, USA

${ }^{4}$ University of Virginia, Charlottesville, Virginia, VA 22904, USA

Correspondence should be addressed to David H. Uttal; duttal@northwestern.edu

Received 31 March 2013; Accepted 22 August 2013

Academic Editor: Jeffrey W. Fagen

Copyright (C) 2013 David H. Uttal et al. This is an open access article distributed under the Creative Commons Attribution License, which permits unrestricted use, distribution, and reproduction in any medium, provided the original work is properly cited.

\begin{abstract}
Three experiments compared performance and transfer among children aged 83-94 months after written or manipulatives instruction on two-digit subtraction. In Experiment la, children learned with manipulatives or with traditional written numerals. All children then completed a written posttest. Experiment $1 \mathrm{~b}$ investigated whether salient or perceptually attractive manipulatives affected transfer. Experiment 2 investigated whether instruction with writing would transfer to a manipulatives-based posttest. Children demonstrated performance gains when the posttest format was identical to the instructed format but failed to demonstrate transfer from the instructed format to an incongruent posttest. The results indicate that the problem in transferring from manipulatives instruction to written assessments stems from a general difficulty in using knowledge gained in one format (e.g., manipulatives) in another format (e.g., writing). Taken together, the results have important implications for research and teaching in early mathematics. Teachers should consider making specific links and alignments between written and manipulatives-based representations of the same problems.
\end{abstract}

\section{Introduction}

The relation between symbolic and concrete representations of mathematical concepts is a recurring theme in mathematics education. On one hand, much of the value of mathematics in human thought stems from its symbolic nature. Symbolic representations of number and mathematical operations allow us to think about abstract mathematical properties and functions independent of a specific quantity or operation. For example, it is possible to know that combining two objects and one object results in three objects without specifying what the objects are. Similarly, we understand that two vehicles traveling at $50 \mathrm{mph}$ move at the same speed regardless of whether both are cars or one is a boat.

On the other hand, much of what we know about mathematics is grounded, at least initially, in our experiences in the world. For example, Lakoff and Núñez [1] suggested that even ostensibly symbolic notions, such as ordinality and logical independence, can be connected, at least metaphorically, to a physical analog (in this case, points that fall on the same line and orthogonal elements in geometry). Concrete thinking pervades numeric judgments, even among individuals who are highly numerically literate. For example, judging that $3>$ 2 requires more time than judging that $5>2$, regardless of whether the problem is posed with objects or symbols [2]. Likewise, visually salient features, such as the physical spacing used to group algebraic terms, influence people's ability to apply a canonical order of mathematical operations (e.g., that multiplication precedes addition) and to subsequently judge the equivalency of simple pairs of equations [3].

Some developmentalists have suggested that young children come to know the world primarily through physical interactions with it [4-9]. On this account, children's mental representations of mathematical ideas are based on concrete experiences, rather than on symbolic, abstract representations of concepts. Consequently, experience with concrete 
representations of mathematical concepts (e.g., relative quantity) is a necessary precursor to having an abstract understanding of the same concept expressed as symbols.

The idea that concrete-based instruction should precede instruction in, or with, symbols has had a significant influence on the design of educational curricula and technologies. One example of this influence is the use of concrete objects, or manipulatives, to teach basic mathematical concepts, including addition and subtraction. Formal manipulatives, such as Cuisenaire Rods, Digi-Blocks, and Base-10 blocks, are objects that are designed specifically to help children learn mathematics. Informal manipulatives are everyday objects, such as pieces of cereal (e.g., Cheerios), paper clips, coins, and play money, that parents or teachers can use to help children learn mathematics [10].

A sizable body of research suggests that the use of both formal and informal manipulatives may facilitate children's development of conceptual knowledge about basic mathematical operations. For example, kindergartners who used a structured set of shaded tiles to learn number sequence, addition, and subtraction outperformed peers who did not use these materials [11]. Fuson and Briars [12] successfully used blocks to teach second and third graders concepts about base-ten concepts. The manipulation of concrete objects helped 9- and 10-year-old fourth graders understand fractions, especially on problems that were difficult to solve without pencil and paper [13]. Middle-school children (4th6th graders) who used informal manipulatives (paper bills and coins) to solve addition and subtraction word problems made fewer conceptual errors than did children who did not use manipulatives [10].

While manipulatives show promise as an instructional tool, questions remain about the ease of transfer between manipulatives and written mathematics problems. Some studies that have demonstrated the advantages of using manipulatives have not demonstrated transfer to written representations $[11,14]$. Other studies that show performance gains while using manipulatives have not even tested transfer to written representations of the same or similar problems.

What obstacles do children face in linking manipulativesbased instruction with written versions of mathematics problems? There are, at least, two possibilities. The first concerns the hypothesized concrete nature of young children's thinking. Perhaps children have trouble transferring their knowledge because (a) they can understand mathematics problems presented in the more concrete format of manipulatives but (b) they have more trouble working in the more symbolic format of writing. This explanation has in fact guided much of the work that has suggested that manipulatives should come before written instruction.

However, there is also a second possibility that is rooted in more general theories of cognition and learning. It is well known that knowledge gained in one context may not transfer to another because of the mismatch between the format in which material was encoded and the format in which it must be recalled $[15,16]$. This consistent finding would support the prediction that transferring knowledge from the written form of mathematics to concrete manipulatives should be just as challenging as transferring in the opposite direction. However, most studies that have investigated the benefits of manipulatives in math education have focused on whether the use of manipulative facilitates knowledge of written solutions and procedures. Much of the research in this field has been motivated by the implicit assumption that concrete representations (in the form of manipulatives) should come before symbols are introduced. As a result, we do not know if the observed difficulty in transfer would occur in the opposite direction: in transferring from writing to manipulatives. Thus, it is not known whether learning a written procedure, for example, improves children's use of and learning from manipulatives. Determining if difficulties in transfer occur in both directions could change the focus of research on manipulatives and written instruction from the particular ordering of materials to whether, when, and how different materials should be introduced. Answering the question about the direction of transfer therefore is a prerequisite to developing forms of instruction that link manipulatives and written instruction.

Young children's well-documented $[17,18]$ difficulty in maintaining a dual representation of a concrete object and its abstract referent may exacerbate the difficulty of general transfer. Children may have difficulty understanding that the manipulatives, particularly if they are familiar objects [19], are not only objects in their own right, but also are intended to be representations of an abstract mathematics concept (e.g., two-digit subtraction). While the connection between a representation and its referent may be perfectly clear to adults, this connection often is less clear to children (e.g., see $[17,20-22])$. Although the two forms of representation may be linked in the mind of the teacher, the two may remain completely separate in the mind of a young child [18].

An often-cited advantage of manipulatives is their procedural flexibility: children can work with them until they find a solution, regardless of whether they follow the standard procedures that would be necessary to obtain a written solution $[11,13]$. However, this procedural flexibility may contribute to difficulties that children encounter in transferring between concrete and written representations. After working with manipulatives, children may find that approaches or procedures that worked with manipulatives cannot be applied to written problems.

Other studies suggest that how symbolic representations are presented to children strongly determines whether or not the symbols are used correctly. It is interesting to note that some researchers and teachers have suggested that manipulatives should be distinctive or otherwise interesting and attractive [19]. However, factors that increase children's attention to the physical properties of symbolic objects, such as bright colors or patterns, can decrease children's understanding of these objects as representations [17, 18, 23-27]. In fact, some recent work has reported that concrete representations can make mathematical concepts more difficult to understand because learners may be distracted by their surface features [28].

In the studies reported here, we investigated the challenges that children face when transferring knowledge from manipulatives to written forms of subtraction problems and vice versa. Children learned to perform two-digit subtraction 


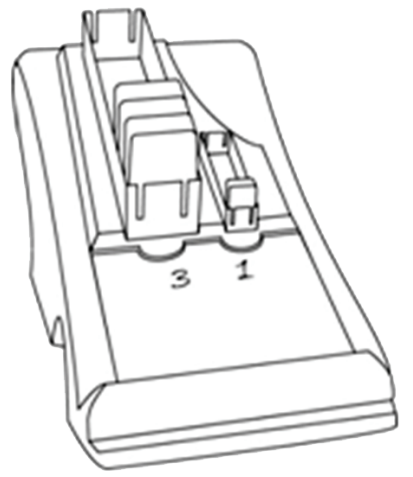

(a)

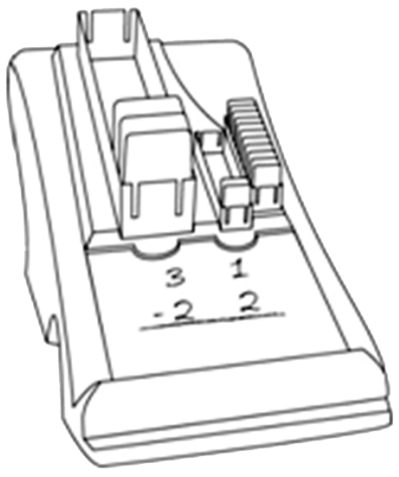

(b)

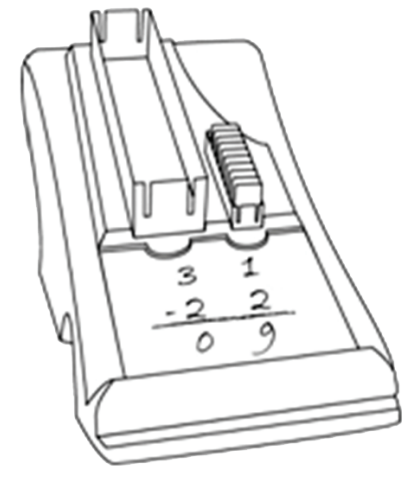

(c)

FIGURE 1: The Digi-Block system, demonstrating the subtraction problem, "31-22." In (a), three blocks of ten on the left side of the counter and a single small block on the right side represent the number "31," which has been recorded below the blocks. In (b), a block of tens has been removed ("borrowed") from the tens position and opened; the ten small blocks inside the borrowed block of ten have been placed to the right side of the counter. The remaining blocks represent the subtrahend (22), which has been recorded beneath the minuend. In (c), two blocks of ten and two single blocks have been removed. The number of remaining blocks (09) has been recorded.

either with the written method or with manipulatives. In Experiment 1a, all children completed a written posttest, while in Experiment 2, the posttest was given with manipulatives. Control groups in both experiments received no instruction. We videotaped children to determine if they used the same solution procedures when problems were presented in manipulative and written formats. We also recorded how long it took for children to solve the problems. In combination, these two studies allowed us to address whether transfer (or lack of transfer) between written and concrete representations occurred in both directions, and how instruction in manipulatives may affect the problem solving strategies children use to solve subtraction problems. In Experiment 1b, we investigated the influences of distinctive manipulatives on children's ability to use them to solve two-digit subtraction problems. In all three experiments, we measured pre- and postinstruction performance and performance on the two practice tests that occurred between the pretest and the posttest.

\section{Experiment 1a}

The goal of Experiment la was to compare the benefits of two forms of instruction to teach two-digit subtraction: the traditional written carryover method and the use of manipulatives. We measured children's performance four times: At an initial pretest, after two instruction periods, and at a final posttest. We measured how much children learned and whether instruction with manipulatives transferred to the more traditional, written problems.

\subsection{Method}

2.1.1. Participants. Thirty children ( 15 males and 15 females) with a mean age of 89.27 months (SD = 2.99 months; range $=83-94$ months) were tested during the summer after first grade. We chose this sample population because they could be expected to have learned simple addition and subtraction in first grade, but would not be likely to know how to solve two-digit subtraction problems. The children were recruited from a database of families who had indicated interest in participating in research. Four additional children were excluded: two asked to leave and two refused to use the manipulatives.

2.1.2. Materials. We used the Digi-Blocks manipulatives system $[29,30]$ to teach two-digit subtraction. We chose this formal manipulative system, in part, because it has the capability to link manipulatives-based and written representations of the same problem. A key component of the Digi-Block design is the nesting of 10 small, sea-green colored plastic blocks (representing units of one) inside a larger container of identical color, called a block of ten. The Digi-Blocks counter holds in place the blocks of ten to the left of the smaller blocks, thus providing a physical instantiation of the concept of place value. The counter also has a small whiteboard that can be used to record a written representation of the problem directly in front of the blocks. Figure 1 illustrates how the Digi-Blocks system would be used to represent a two-digit subtraction problem.

We used a digital camera to record children's problem solving procedures on all tests. We later coded the videotapes to indicate when children started and stopped the subtraction process and whether children started the subtraction process in the ones column or in the tens column. In addition, we noted when a child's subtraction procedure could not be clearly identified as either starting in the ones or starting in the tens column.

2.1.3. Procedure. Children were assigned randomly to one of three groups: written instruction, manipulatives instruction, or control. Children in all groups completed the same, written pretest and posttest. The groups differed in terms of (a) whether they received instruction, (b) the form of the instruction, and (c) how the effects of instruction were assessed. The design of Experiment 1a is shown in Table 1. 
TABLE 1: Experiment 1 design.

\begin{tabular}{llcccr}
\hline & $n$ & Pretest & Practice test 1 & Practice test 2 & Posttest \\
\hline Control (no instruction) & 10 & Written & Written & Written & Written \\
Written instruction & 10 & Written & Written & Written \\
Manipulatives instruction & 10 & Written & Manipulatives & Manipulatives & Written \\
\hline
\end{tabular}

The experiment took place on two consecutive days. On day 1 , children completed a written pretest, which provided baseline information of their knowledge of two-digit subtraction. The children were given two sample problems before the pretest to ensure that they understood the requirements of the problem. If they were not able to complete the sample problems, the experimenter provided the correct answers but did not provide explicit instruction on problem solutions. After the pretest, children in the written and manipulatives instruction groups received instruction and took a test (Practice Test 1) to assess the effects of that instruction.

Written Instruction Group. After the pretest, the experimenter began instruction in the written method. The experimenter demonstrated the written carryover procedure of doubledigit subtraction: starting in the ones column, borrowing from the tens position if needed, carrying over to the ones position, and subtracting the numbers in the ones and tens columns. In two sample problems, the experimenter demonstrated how to start in the ones column, borrow from the tens position if needed, carry over to the ones position, and subtract the numbers in the ones and tens position to arrive at a solution. The children completed four additional problems on their own, with the experimenter providing corrective feedback as needed. The children then completed the first practice test, which for this group was simply a set of seven written two-digit subtraction problems. The same procedure was used on the second day for the instruction period and the second practice test.

Manipulatives Instruction Group. Children in the manipulatives instruction group took the same written pretest and posttest as the written instruction group did. However, during instruction, the practice tests were administered using manipulatives. The manipulatives instruction was based on the protocol specified in the Digi-Block's "Companion to Everyday Mathematics," Grades 1 and 2 [29-31], which is a supplement to the Everyday Mathematics curriculum. The experimenter demonstrated the use of manipulatives with the same subtraction problem that was used to demonstrate the carryover method in the written instruction condition. As in the written instruction condition, the protocol for the manipulatives instruction emphasized that children should start the subtraction procedure in the ones column, by counting the single blocks. The subtraction problem, "31-22," is illustrated in Figure 1. The instructor first demonstrated that she could not remove two small blocks from the ones column when only one small block was available. She then "borrowed" a block of ten by opening it and moving its ten small blocks to the right of the ones column. Next, the experimenter subtracted (removed) the two small blocks and the remaining two blocks of ten from counter. Finally, she pointed out that there were 9 small blocks left.

After observing the two example problems, the children practiced on four subtraction problems. The experimenter provided corrective feedback when children made a mistake and encouraged them to concentrate on the steps they used to solve the problems. After the practice problems, the children completed the first practice test. Children were instructed to use the Digi-Blocks to answer each question and to move on to the next problem if they could not answer a particular question. The same protocol was used on the second day.

Control Group. Children in the control group took the same tests as children in the written instruction group but received no instruction. Control group children were allowed to use any method (counting on fingers, guessing, using the carryover method, etc.) to solve the problems.

Posttest. Children in all groups completed a written sevenproblem posttest at the end of the second day. The experimenter gave no explicit instruction prior to or during the posttest. The children were allowed to solve the problems using any method they wished, with the exception that no group had access to the blocks. After they completed the posttest, the experimenter asked children in the manipulatives condition if they had thought about the blocks while they completed the written posttest.

Content of Assessments. The subtraction problems used in the four tests (the pretest, the two practice tests, and the posttest) were selected from Everyday Mathematics [32], a curriculum that was used in many of the school districts from which the participants were selected. In each problem, a twodigit number was to be subtracted from another two-digit number. In their written format, many problems required the carryover method to solve, that is, "borrowing" from the tens unit to complete subtraction in the ones unit. Problems varied in difficulty, and more challenging problems were balanced across the four sets of problems. Any problem set could be assigned to any of the measures (pretest, practice tests 1 and 2 , or posttest).

\subsection{Results and Discussion}

2.2.1. Effect of Type of Instruction on Test Performance. Figure 2 shows mean performance for the different groups on the 4 different measures, that is, the pretest, the two practice tests, and the posttest. The figure reveals several important results. First, as we expected, the performance of the three groups did not differ significantly at pretest. Second, both the written instruction and manipulatives instruction groups improved their performance on practice tests 1 and 2 , relative to pretest. Third, there were important differences 


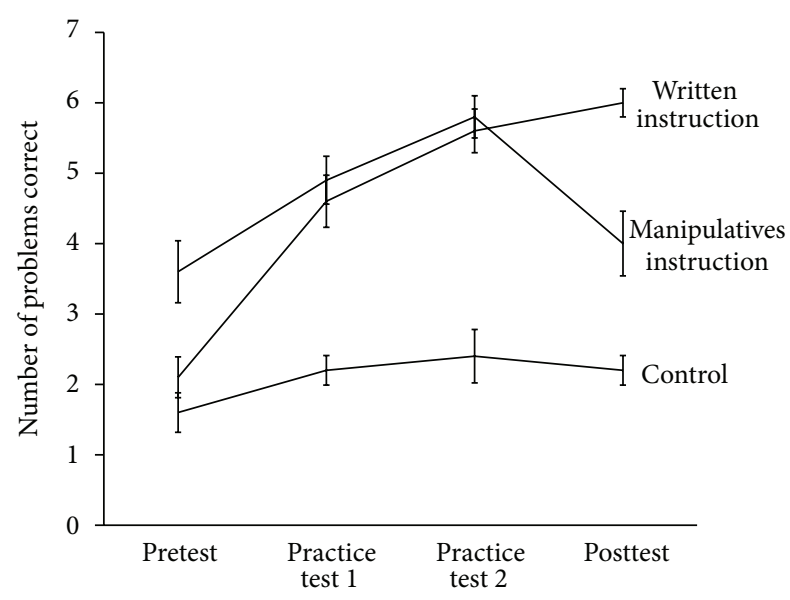

Figure 2: Number of problems correct, by condition, across four tests in Experiment 1.

in performance between the two groups on the posttest. The performance of the written instruction group changed a little from practice test 2 to posttest; this group clearly demonstrated what they had learned on the posttest. In contrast, the performance of the manipulatives instruction group decreased precipitously from practice test 2 to posttest. In fact, the manipulatives instruction group performed about the same on the posttest and pretest. The performance of the control group did not change significantly across the four tests.

These results were confirmed in a one-way analysis of covariance (ANCOVA), which found a significant effect of instruction on posttest scores, $F(2,26)=17.84, p<.001$, and $\eta_{p}^{2}=.58$. The ANCOVA was chosen as the appropriate test for this preposttest control educational experiment, as recommended by Dugard and Todman [33]. The covariate, pretest scores, was significant, $F(1,26)=36.53, p<$ .001 , and $\eta_{p}^{2}=.58$. On posttest, children in the written instruction group had the largest adjusted mean scores $(M=$ $6.00 ; \mathrm{SD}=1.25)$, followed by the control group $(M=$ $4.20 ; \mathrm{SD}=2.1$ ) and the manipulatives instruction group $(M=2.20 ; \mathrm{SD}=1.99)$. Pairwise comparisons revealed that the written instructions group performed significantly better than the manipulatives instruction group, $p<.001$, which, in turn, performed significantly better than the control, $p<$ .001 . On average, the written instruction group improved by 3.9 ( $\mathrm{SD}=1.73$ ) (out of 7 ) problems from pretest to posttest, and this change score differed significantly from $0, t(9)=$ 7.13, $p<.001$. In contrast, the manipulatives instruction $(M=0.4 ; \mathrm{SD}=1.35)$ and control groups $(M=0.6 ; \mathrm{SD}=$ $1.35)$ barely improved at all, and the change scores did not differ significantly from $0, t s(9)<1.41, p s>.19$.

\subsubsection{Effect of Type of Instruction on Time to Solve Problems.} We measured solution time on the subtraction problems for each test. For the written instruction and control groups, time to complete each test was relatively stable over the four tests. In contrast, the manipulatives instruction group took much longer to complete the two practice tests, which were

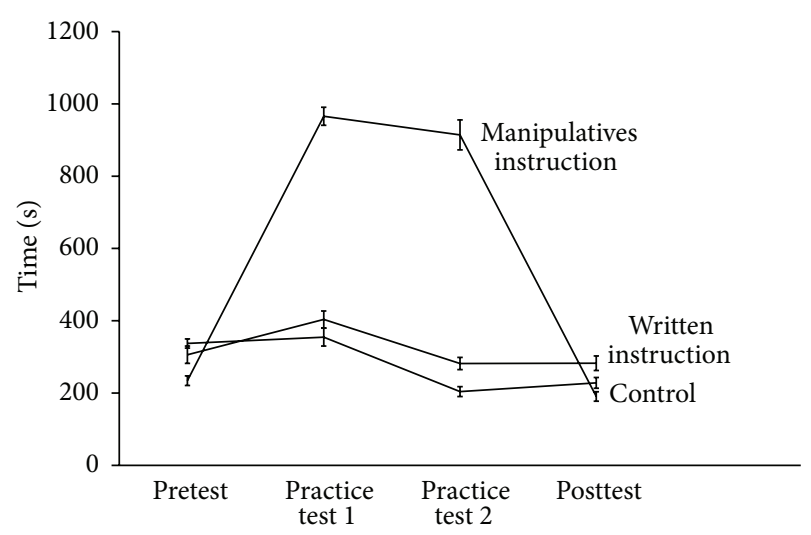

FIGURE 3: Mean time to complete tests, by condition, in Experiment 1 .

given in manipulatives format, than they did to complete the pre- and posttests, which were given in the written format. Figure 3 shows mean time, in seconds, for participants in each condition to complete each of the four tests (pretest, practice test 1, practice test 2, and posttest) in Experiment 1. A repeated measures ANOVA, with test type (pretest, practice test 1, practice test 2 , and posttest) as the within-subjects factor and condition as a between subjects factor, found a significant effect of test type, $F(3,81)=59.51, p>.001$, and $\eta_{p}^{2}=.69$, and a significant interaction between test type and condition, $F(6,81)=45.29, p<.01$, and $\eta_{p}^{2}=.77$.

2.2.3. Effect of Type of Instruction on Subtraction Procedures. Did the type of instruction (written, manipulatives, or no instruction) affect children's use of different procedures for two-digit subtraction? To investigate this question, we videotaped children as they solved subtraction problems and coded across four tests (pretest, practice tests 1 and 2, and posttest) the frequency with which children attempted to solve subtraction problems by starting in the ones column or by starting in the tens column. (Note that some children were coded as using neither strategy. This code was used when children did not attempt to solve the problem (and hence did not use a strategy) or when the coder was unable to tell which strategy the child had used). A univariate ANOVA found a significant effect of type of instruction on how often children started their solutions in the ones column across four tests, $F(2,27)=22.23, p<.001$, and $\eta_{p}^{2}=.62$. Furthermore, there was a significant positive correlation between starting in the ones column on the posttest and posttest performance, $r(28)=.62, p<.001$; those children who started in the ones column performed substantially better than those who did not. A Fisher's least-significant difference post hoc test revealed that children in the written instruction condition started in the ones column significantly more often than children in the manipulatives condition, $p<.02$, and significantly more often than children in the control group, $p<.001$.

Next, we investigated how children used the starting-inthe-ones procedure over the course of instruction, by test. At pretest, there were no significant differences among the three 


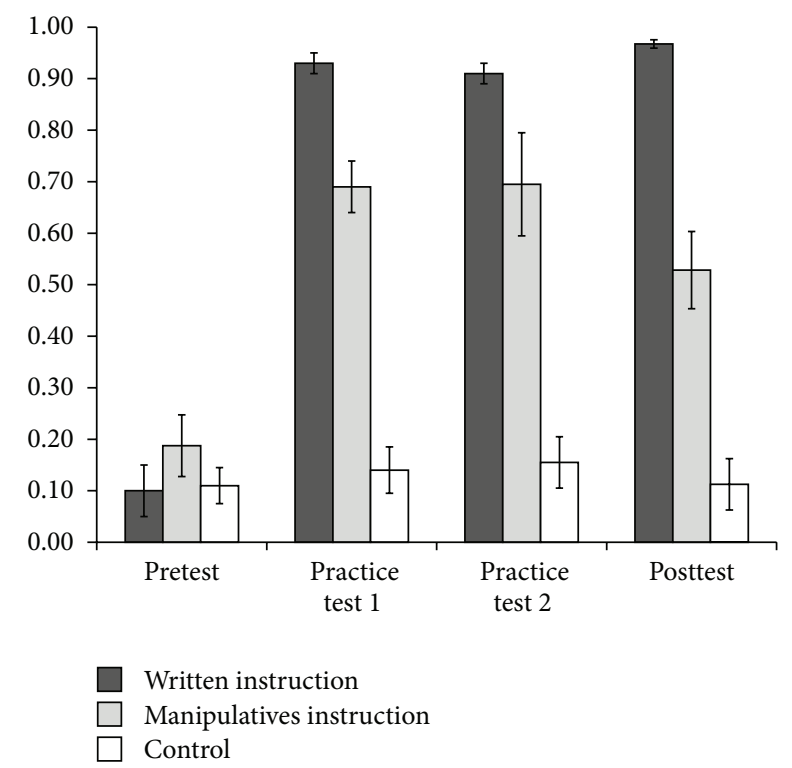

FIGURE 4: Use of starting-in-the-ones column by test and condition, in Experiment 1.

conditions in use of the starting-in-the-ones procedure, as demonstrated by a univariate ANOVA, $F(2,27)=.24, p=$ .79 , and $\eta_{p}^{2}=.02$. Thus, before instruction the three groups were equally matched in their tendency to start subtraction in the ones column. The effect of instruction on children's use of the procedure began to emerge after the first practice session. A univariate ANOVA, $F(2,27)=21.99, p<.001$, and $\eta_{p}^{2}=.62$ demonstrated a significant effect of condition on children's use of the starting-in-the-ones procedure on the practice test 1. A post hoc Fisher's least-significant difference contrast demonstrated that children in the written condition started in the ones column marginally more often than children in the manipulatives, $p=.06$. At the second practice test, the contribution of instruction was still significant, $F(2,27)=$ $16.99, p<.001, \eta_{p}^{2}=.58$. As shown in Figure 4, the written instructions group started in the ones more frequently than the manipulatives instruction group did, although this difference did not reach significance as demonstrated by a post hoc Fisher's least-significant difference contrast, $p=$ .12. The differences, by condition, in the use of the startingin-the-ones procedure remained significant at posttest, as demonstrated by a univariate ANOVA, $F(2,27)=16.35, p<$ .001. A post hoc Fisher's least-significant difference contrast revealed that children in the written condition used the starting-in-the-ones procedure significantly more than did children in the manipulatives condition, $p<.007$.

Taken together, these analyses indicate that different methods of instruction led children to solve the problems differently. Although all children were taught to start in the ones column, children in the manipulatives condition did so significantly less often than did children in the written condition. This pattern is not surprising, as instruction in the written method tends to emphasize a fixed, sequenced procedure that starts in the ones column. Manipulativesbased instruction affords a more flexible approach to solving these problems that allows for alternative approaches, such as starting in the tens column. Thus, even though the manipulatives instruction group was explicitly instructed to start in the ones column, they followed this advice significantly less often than the written instructions group did.

In summary, Experiment la demonstrates that the children in both instructional groups (written and manipulatives) learned to perform two-digit subtraction using the respective instructional format. However, children who were instructed with manipulatives had difficulty solving the written problems without the blocks.

\section{Experiment 1b}

In this experiment we investigated the effects of distinctive manipulatives on children's learning of two-digit subtractions. It is often suggested that manipulatives should be interesting or physically attractive, to garner children's attention and interest [19]. However, the dual-representation hypothesis leads to a somewhat different perspective: highly distinctive manipulatives may be distracting and lead children to focus more on the manipulatives themselves rather than on the mathematical concepts that the teacher is attempting to communicate. In Experiment $1 \mathrm{~b}$ we investigated this idea. We presented a new group of children with a visually distinctive set of manipulatives and compared their performance and the time it took to solve the problems to that of the manipulativesinstructed group in Experiment 1 (which we label the standard manipulatives instruction group in this experiment).

To make a highly distinctive set of manipulatives, we used nontoxic permanent markers to add different colors and patterns to each Digi-Block. Each block had one of five different base colors (e.g., blue or red) and a unique pattern or drawing, such as swirls or polka dots. Thus, the distinctive set maintained the base-ten relations of the original set; the only difference was that we added colors and patterns to each block.

3.1. Participants. Nineteen children (11 girls and 8 boys) participated. Their mean age was 88.58 months $(\mathrm{SD}=$ 2.65 ), range $=83-94$ months. As before, all children were tested during the summer break after they had completed first grade and were recruited from an existing database of families. Nine children were assigned to see the standard manipulatives and 10 children were assigned to distinctive manipulatives. The children in the standard group were the same children who comprised the manipulatives instruction group in Experiment 1.

3.2. Procedure. As in Experiment la, each child completed 28 double-digit subtraction problems and participated for two sessions that lasted approximately one hour each, one day apart. The procedures were identical to those of Experiment la; the only difference was in whether the children saw the standard or distinctive manipulatives.

\subsection{Results and Discussion}

3.3.1. Effect of Block Alteration on Performance. At pretest, the difference in performance between the distinctive blocks 
group $(M=2.40 ; \mathrm{SD}=1.90)$ and standard blocks group $(M=4.71 ; \mathrm{SD}=2.11)$ was not significant, $P=.13$. We examined the role that manipulative block type (visually distinctive versus standard) played on children's scores and on their behaviors with the blocks. We conducted a oneway ANCOVA, investigating the effect of block alteration on posttest performance and covarying pretest performance. The effect of the covariate, which was pretest score, was significant, $F(1,16)=26.97, p<.001$, and $\eta_{p}^{2}=.63$. There was no significant effect of surface alteration, $F(1,16)=$ 2.02, $p=.17$, and $\eta_{p}^{2}=.112$. At posttest, the performance of children who used nonaltered standard blocks $(M=$ $3.67 ; \mathrm{SD}=2.87)$ was higher than that of children who used altered distinctive blocks $(M=3.00 ; \mathrm{SD}=2.21)$, although this difference did not reach statistical significance.

3.3.2. Effect of Block Alteration on Subtraction Strategies. We conducted a one-way ANCOVA investigating the effect of block alteration on subtraction strategies. There was a significant effect of block alteration, $F(1,16)=5.69, p=$ .03 , and $\eta_{p}^{2}=.25$. Children in the standard block condition started in the ones column significantly more $(M=.60 ; \mathrm{SD}=$ .34) than children in the distinctive block condition $\operatorname{did}(M=$ .29003 ; SD $=.21$ ). Because children in both manipulatives instruction groups (standard and distinctive) were taught to start in the ones column, this result suggests that children who received the plain, standard blocks followed these instructions to a greater extent than children who received the visually distinctive blocks did.

\subsubsection{Effect of Block Alteration on Mathematical Behaviors.} We also observed children's actions with the blocks, coding whether the behaviors were directed at solving the problem or were nonmathematical, such as building towers or sorting by color. Two raters independently evaluated the videotapes of each child's participation, and interrater reliability was very high (Cohen's kappa $=.85, p<.001$ ). For each of the seven problems on the first and second test sets (14 problems in total), we assessed whether any children used the blocks in a nonmathematical way during the problem. Only one participant in the standard manipulatives instruction group used the blocks in a nonmathematical way while completing the first and second test sets. In contrast, seven out of 10 participants in the distinctive manipulatives instruction group did so on a number of problems, ranging from 1 to 13 problems (Mann-Whitney $U=23.00, p<.05$ ). These results indicate that, while the blocks may be more visually appealing to children, the additional colors and patterns on the distinctive manipulatives distracted children from solving the math problems, possibly making it more difficult for children to build a connection between the manipulatives and the written representations. Thus, we found that visually distinctive, compared with standard, manipulatives tended to detract from mathematics instruction. Differences in how children treated the two sets of manipulatives may have important implications for the design of manipulative systems.
3.3.4. Effect of Surface Features on Time to Solve Problems. The surface alteration of the blocks did not significantly increase the time children spent on problems. A repeated measures ANOVA, with test type (pretest, practice test 1, practice test 2 , posttest) as the within-subjects factor and type of block (distinctive versus standard) as the between-subjects factor found a significant effect of test type, $F(3,51)=136.48, p>$ .001 , and $\eta_{p}^{2}=.89$, but no significant interaction between test and type of blocks, $p=.89, \eta_{p}^{2}=.45$.

In summary, we did not find a significant difference between the performances of the distinctive and standard manipulatives instruction groups. In part this may reflect rather large variability, particularly in the distinctive manipulatives instruction group. However, we did find two potentially important ways in which the distinctive manipulatives led to differences in children's behavior. First, children who learned with the distinctive manipulatives were even less likely than the standard manipulatives instruction group to begin their calculations in the ones column. Second, children who learned with the distinctive manipulatives used them more often in mathematically irrelevant ways, such as building towers. Taken together, these results suggest that highly distinctive manipulatives could make it harder for children to focus on the learning of mathematics.

\section{Experiment 2}

In Experiment $1 \mathrm{a}$ and $1 \mathrm{~b}$, children successfully learned two-digit subtraction from both written and manipulatives instruction. However, children who received manipulatives instruction had difficulty transferring what they learned to a written posttest. Was the manipulatives instruction group's failure to transfer related to the mode of instruction they received, or is it indicative of a more general difficulty in transferring from one mode of instruction to a different mode of testing? We investigate this question in Experiment 2, in which the posttest was administered in a manipulatives format, rather than in a written format. Thus, in Experiment 2 , the children in the written instruction group experienced a mismatch between the form of instruction and the form of the posttest. If these children now do poorly on the posttest, it would suggest that the problem we observed in Experiment la was due to the general need to switch modalities, rather than the specific ordering of manipulatives followed by written instructions that was followed in Experiment 1a. The design of Experiment 2 is summarized in Table 2.

\subsection{Method}

4.1.1. Participants. Fifteen girls and 15 boys participated in the study ( $M$ age $=89.4$; SD $=3.61$ months; range $=83-$ 96). All children were tested during the summer break after they had completed first grade. Recruitment procedures were identical to those used in Experiment 1.

4.1.2. Materials and Procedures. Children were randomly assigned to the written instruction, manipulatives instruction, or control group. The design of Experiment 2 was very similar to that of Experiment 1a; the only difference was the 
TABLE 2: Experiment 2 design.

\begin{tabular}{llcccc}
\hline & $n$ & Pretest & Practice test 1 & Practice test 2 & Posttest \\
\hline Control & 10 & Written & None & None & Manipulatives \\
Written instruction & 10 & Written & Written & Mritten & Manipulatives \\
Manipulatives instruction & 10 & Written & Manipulatives & Manipulatives & Manipulatives \\
\hline
\end{tabular}

Note: In Experiment 1, children who received no instruction did not improve their performance after completing two practice tests. Consequently, in Experiment 2 children who received no instruction were given only the pretest.

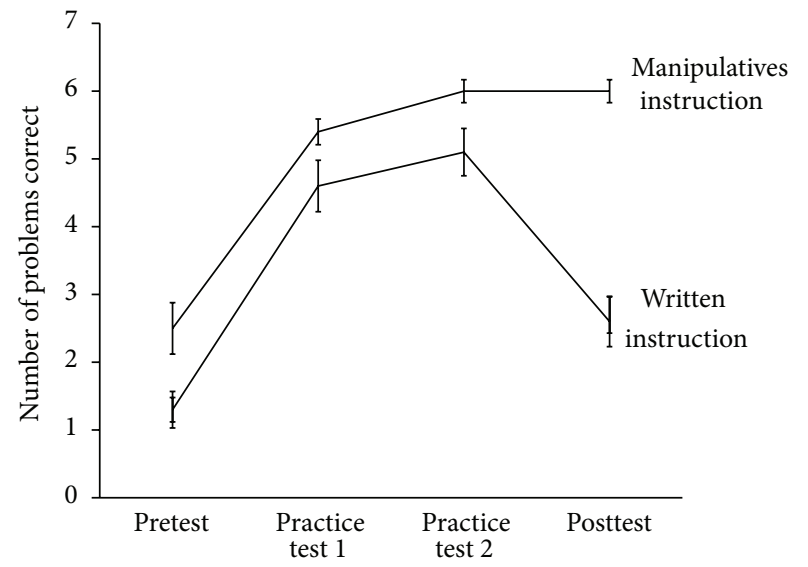

FIGURE 5: Number of problems correct, by condition, across four tests in Experiment 2.

format of the posttest. In Experiment 2, children in all three groups completed the posttest using the manipulatives.

Introducing the manipulatives at the posttest did require some additional instructions for children in the written instructions group. Prior to the posttest, the experimenter introduced the Digi-Blocks to these children by pointing out the blocks of ten and the single blocks, adding, "Some people find these blocks helpful in answering the types of problems we have been working on." Participants were then asked to solve the problems using the Digi-Blocks and were provided with a pencil to record their answer after they had reached a solution. As in Experiment 1a, no procedural instruction or assistance was given during the posttest.

The procedure for the control group was similar to that of Experiment 1a, but two differences should be noted. First, the control group in Experiment 2 completed only the pretest and posttest, all on the same day. The lack of learning across the two days in the control group in Experiment la suggested that it was not necessary to have the control group complete all four assessments. Second, in contrast to the procedures for the written and manipulatives instruction group, the control group did not use the manipulatives counter at posttest (see Figure 1) but instead solved the problems only with the blocks. We reasoned that providing the counter might constrain children's solutions or, in essence, provide implicit instruction on the use of the blocks.

\subsection{Results and Discussion}

4.2.1. Effect of Instruction on Performance. The results clearly indicate that the failure of transfer also holds when children are asked to apply what they have learned from written instruction to using manipulatives. Figure 5 shows performance means across four tests for the two instructed groups. The performance of the control group is not shown because they completed only the pretest and posttest, and their performance on these assessments was similar to that of the written instruction group. The written instruction and manipulatives instruction groups both improved their performance on practice tests 1 and 2, relative to their respective pretest performance. As in Experiment 1a, both instruction groups learned from their respective forms of instruction. At posttest, children in the manipulatives instruction group, who had learned with the manipulatives throughout, had the largest adjusted mean scores $(M=6.00 ; \mathrm{SD}=1.05)$, followed by the control group $(M=2.70 ; \mathrm{SD}=1.70)$ and the written instructions group $(M=2.60 ; \mathrm{SD}=$ 2.32). Unlike Experiment 1a, in which the performance of the manipulatives group decreased from practice test 2 to posttest, there was no significant difference between the performance of the manipulatives instruction group at posttest compared to practice test 2 . This demonstrates that children instructed in the manipulatives method were able to apply what they had learned during instruction to the posttest. In contrast, the performance of the written instructions group plummeted from practice test 2 to the posttest, suggesting that they had difficulty transferring what they had learned during instruction to the manipulatives that were used at the posttest.

Thus the pattern of results was very similar to that of Experiment la in that the group that used the same materials throughout performed well, whereas the group that switched to a different form of representation at posttest did poorly. In Experiment 2, however, it was the manipulatives instruction group that had the same materials throughout, whereas in Experiment la it had been the written instructions group. This result clearly indicates that whether posttest performance is good or poor is not dependent on which learning materials were given but on whether there was a need to transfer from one (e.g., manipulatives) to the other (e.g., writing).

We conducted a one-way ANCOVA to investigate the effect of instruction on posttest performance. As in Experiment 1 , the covariate was pretest performance. There was a significant effect of instruction on posttest scores, $F(2,26)=$ 9.38, $p<.001$, and $\eta_{p}^{2}=.42$. The magnitude of this difference, although substantial, was somewhat smaller than the effect of instruction observed in Experiment 1a. The effect of the covariate, pretest, was nonsignificant, $F(1,26)=$ $1.06, p=.32$, and $\eta_{p}^{2}=.04$. Note that in contrast to Experiment 1, the pretest and posttest in Experiment 2 were 


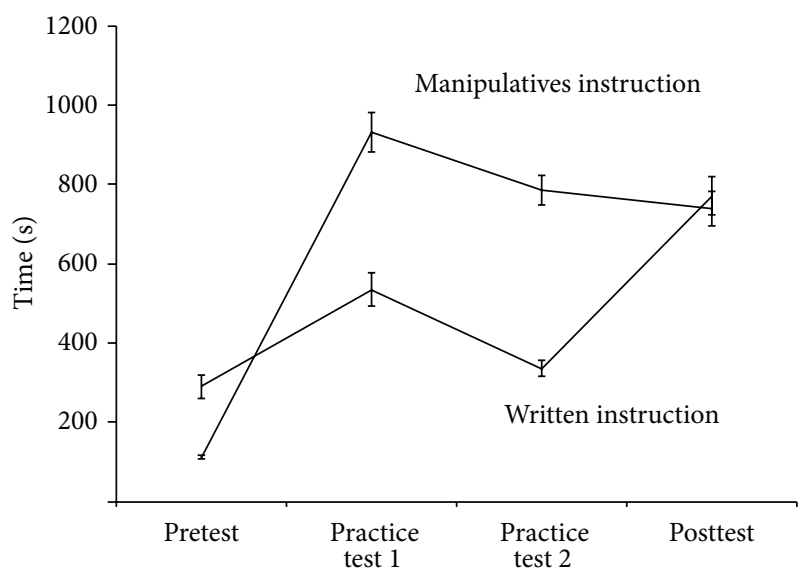

Figure 6: Mean time to complete tests, by condition, in Experiment 2.

presented in different formats. This difference may help to explain the lack of an effect of the covariate (pretest) on posttest performance.

The manipulatives instruction group improved on average by 3.5 problems, which differs significantly from $0, t(9)=$ $4.34, p<.01$. The written instructions group $(M=$ $1.30 ; \mathrm{SD}=2.50)$ did not improve significantly from pretest to posttest, $t(9)=1.65, p>.13$. The control group $(M=$ $1.4 ; \mathrm{SD}=1.65)$ did improve significantly from pretest to posttest, $t(9)=2.70, p<.05$.

4.2.2. Effect of Instruction on Time to Solve Problems. Figure 6 shows mean time, in seconds, for participants in the two instructed conditions to complete each of the four tests (pretest, practice test 1, practice test 2, and posttest) in Experiment 2 (as noted in the Method section, the control group completed only the pre- and posttest). A repeated measures ANOVA, with test type (pretest, practice test 1, practice test 2 , and posttest) as the within-subjects factor and condition as the between-subjects factor, found a significant effect of test type, $F(3,54)=39.84, p>.001$, and $\eta_{p}^{2}=$ .69 , and a significant interaction between test type and condition, $F(3,54)=14.76, p>.001$, and $\eta_{p}^{2}=.45$. The control group took about the same amount of time to solve the problems on the pre- and posttests. For the written instructions group, time to complete each of the four tests was relatively stable across four tests. The manipulatives instruction group took longer to complete practice test 1 than any of the other tests in Experiment 2. The results clearly reflect that using the manipulatives takes more time than using writing alone. The manipulatives instruction group spent almost twice as much time on the two practice tests as the written instructions group. However, at posttest, when all groups used manipulatives, the manipulatives and written instruction group took almost exactly the same amount of time.

Experiment 2 allows us to separate the effects of time to learn with manipulatives from those of time required to solve the problems with manipulatives, once the information

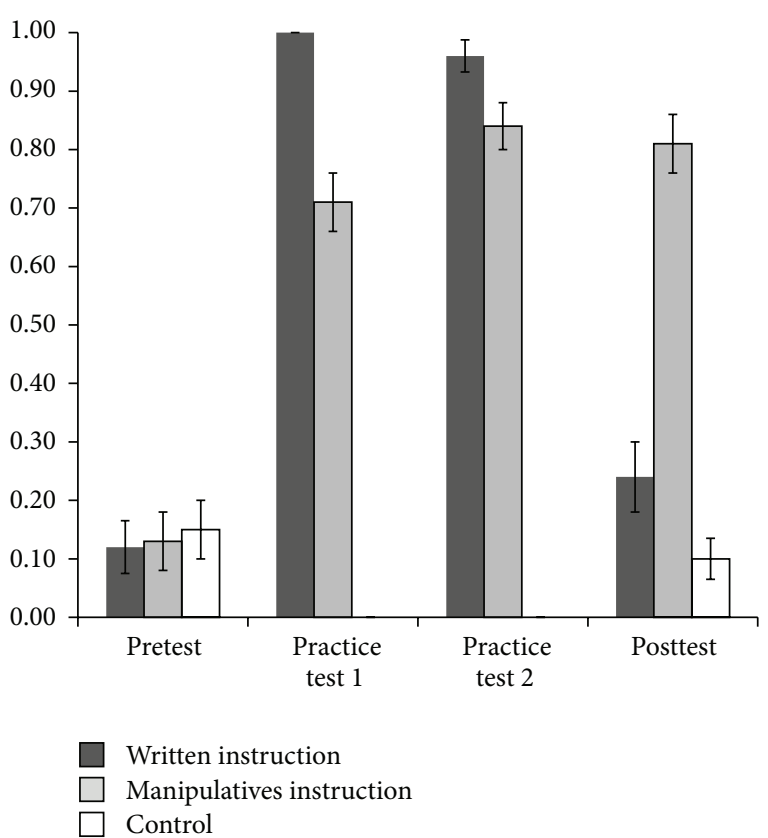

FIGURE 7: Use of starting-in-the-ones strategy by test and condition, in Experiment 2.

is learned. Did children in the manipulatives condition get faster with the blocks as they mastered the mathematical content? If so, then we would expect to see small differences between the written instructions group and the manipulatives instruction group in time to finish the posttest. Using a univariate ANOVA, we compared the time to solve the posttest for the written and manipulatives instruction groups only, covarying for time to solve the pretest. There were no significant differences between the written and manipulatives instruction groups in time to solve the posttest, $F(1,17)=$ $.00, p=.99$, and $\eta_{p}^{2}=.00$. The covariate was also nonsignificant, $F(1,17)=.11, p=.75$, and $\eta_{p}^{2}=.01$. Thus, although children required more time to learn with manipulatives, they were able to solve the posttest problems as quickly as those children who had learned with the written method.

4.2.3. Effect of Type of Instruction on Subtraction Strategies. For the two instructed conditions, we coded videotapes for starting the subtraction process in the ones column across four tests. We coded the control group's subtraction procedures for the pre- and posttest. Figure 7 shows means for starting in the ones column, across four tests. The results of a univariate ANOVA showed a significant effect of condition on children's use of the starting-in-the-ones procedure across four tests, $F(2,27)=31.57, p<.001$, and $\eta_{p}^{2}=$ .70. As in Experiment 1, there was a significant positive correlation between posttest performance and starting in the ones column on the posttest, $r(28)=.51, p<.001$. Thus, as in Experiment 1, children who started in the ones column, as instructed, performed substantially better than those who did not. 
To further investigate children's subtraction strategies over the course of instruction, we analyzed the use of the starting-in-the-ones strategy by test. The three groups were equally matched in their tendency to start subtraction in the ones column on the pretest, as demonstrated by a univariate ANOVA, $F(1,27)=.02, p=.98$, and $\eta_{p}^{2}=.002$, and shown in Figure 7. On practice test 1 , condition made a significant positive contribution to use of the starting-in-theones procedure, $F(1,18)=7.5, p=.01$, and $\eta_{p}^{2}=.29$, with the written instruction group starting in the ones column significantly more often than the manipulatives instruction group (recall that the control group did not complete practice tests 1 or 2 ). On practice test 2, there were no significant differences between the written instruction group and the manipulatives instruction group, $F(1,18)=1.55, p=$ .23 , and $\eta_{p}^{2}=.08$. However, at posttest, the manipulatives instruction group used the starting-in-the-ones procedure significantly more than the written instruction condition on posttest, $F(2,27)=14.75, p<.001$, and $\eta_{p}^{2}=.52$. Children in the manipulatives instruction group scored significantly higher than those in the control group, $p<.001$, and significantly higher than those in the written instructions condition, $p<.001$, as demonstrated by Fisher's leastsignificant difference post hoc tests. In Experiment 2, children in the written instructions group were much more likely to use a strategy at posttest that was different from the one they had successfully used during the practice tests. In essence, this result shows that children followed the procedure that they were taught as long as the format of the posttest matched the format in which they were taught. As long as children continued to solve problems in the format in which they were taught, their strategies remained consistent. However, as soon as children were required to change problem format, they became much more likely to adopt a different strategy.

\section{General Discussion}

A common approach that has guided much of the research on manipulatives has been to focus on whether manipulativesbased instruction is better, worse, or as good as traditional written methods of instruction. Some research [11, 12, 34] has emphasized the benefits of manipulatives, whereas other investigations have raised criticisms of their use $[14,35,36]$. Our research may contribute to redirecting the focus of this debate. We found no inherent advantage either for manipulatives or for the written method in terms of the number of test items answered correctly. In fact, children instructed in either format were able to demonstrate very high levels of competence within a short period of instruction, as long as they were tested in the same format in which they were instructed. Performance deteriorated only when children were required to solve problems in the noninstructed format. This finding may explain why research that focuses on pitting the two modes of instruction against one another has often yielded mixed results, with manipulatives sometimes, but not always, appearing to be an improvement upon standard written instruction.
5.1. Differences between Manipulatives and Written Instruction. The present work also provides insight into why transfer did not occur, either from manipulatives to writing in Experiment 1 or from writing to manipulatives in Experiment 2. Anecdotal evidence suggests that many children did not think of the manipulatives and written problems as being related to each other. For example, at the end of Experiment 1, we sometimes asked children whether they had thought about the blocks when completing the written pretest. Almost all children answered "no," even though we had suggested to them that thinking about what they had learned with the blocks might help them in solving the written pretest. In combination with their poor performance on the posttest, this suggests that their solutions to the written problems were not influenced by their work with the manipulatives. This explanation is consistent with previous work that has demonstrated that children often have difficulty seeing the relationships between concrete and abstract representations of the same problem [14, 18, 22, 28].

Second, at a more specific level, the results also suggest that manipulatives and written instruction may encourage children to learn different procedures that are not always equally accessible or mutually reinforcing. In the first and second practice tests of Experiments 1a and 2, some children in the manipulatives condition solved some problems without starting in the ones column, even though they had been taught to do so. Rather than starting in the ones column, these children subtracted the blocks of ten before borrowing from the tens position and subtracting the blocks in the ones position. Although this method yielded the correct answers when the children used manipulatives, the same procedure often would not generate the correct solution on written problems. Manipulatives permit children to solve two-digit subtraction problems in multiple ways. This flexibility may carry advantages in terms of conceptual understanding, but it may also carry some disadvantages. For example, it may make it more difficult for children trained in manipulatives instruction to discover the correct order in which procedural operations must be performed in the written method.

5.2. Time to Complete Problems. During the practice tests Experiments of 1 and 2, children took twice as long to learn and solve problems with manipulatives as they did with the written method. Much of the additional time required during manipulatives instruction can be accounted for by the time needed to physically manipulate the blocks. In combination, the results of Experiments 1 and 2 rule out the possibility that the manipulatives instruction groups got substantially faster as they became more familiar with blocks. In both experiments, the children were much slower when they used manipulatives than when they used writing. Thus the decrease in time in Experiment 1 at the posttest represents the switch to writing, rather than a decrease in the time needed to use the manipulatives. The reduced amount of time required to complete the problems in writing is one of the advantages of using numeral and mathematical symbols that could accrue once children have established adequate groundings to allow them to use the symbols in a meaningful way. 
5.3. Should Manipulatives Be Visually Distinctive? Several authors (e.g., [19]) have noted that American teachers often believe that manipulatives should be attractive, interesting, or visually distinctive to enhance children's interest in them. Likewise, teachers may also believe in the value of varying manipulatives, using a host of different manipulatives (both formal and informal) to avoid boredom. However, the present results and those of other researchers (e.g., $[10,27])$ indicate that using distinctive objects or those that have been used in other contexts (e.g., money, Cheerios, paper clips, etc.) may actually engender additional difficulties with transfer of knowledge, particularly to written procedures. In the present study, we found that children were more likely to treat the visually distinctive manipulatives in nonmathematical ways, such as building towers or playing other games with the blocks. Manipulatives may be a case in which less is more; decreasing children's interest in the manipulatives as objects in their own right may help the children to focus more on the mathematical information that is being taught [27].

5.4. Implications for Instruction. The results clearly indicate that children can learn quickly and well from either manipulatives or from the written method. However, regardless of the format in which they learn, they have trouble transferring their knowledge to the other format. Here we consider two questions arising from our results that are highly relevant for instruction.

The first question is whether it makes sense to continue to use manipulatives. We demonstrated that manipulativesbased instruction may not transfer to written assessments, and written assessments are typically how students will be required to express their knowledge. As mentioned earlier, part of the advantage of mathematics is its symbolic nature, and using the written system leads to far faster solutions. Given these issues, why should teachers use manipulatives at all? We believe that the answer is that manipulatives can have very important advantages in situations that differ from those used in the present experiment. Here our goal was to teach a specific mathematical procedure, and in these situations, written instruction may be faster and more efficient. However, in other situations, where the goal involves conceptual understanding, the use of manipulatives may lead to a more grounded and deeper understanding. Both procedural and conceptual knowledge are required for longterm success in mathematics, and thus both manipulativesand written-based instruction will continue to be useful.

The second question is how we can help children to connect what they learn from manipulatives and from written instruction. Our results, and those of others, suggest that this serial introduction of manipulative and written instruction (regardless of the order in which the materials are introduced) carries the risk of children failing to connect the two. It may be worthwhile to consider whether children can learn about, and hence associate, both forms of representation at the same time.

We suggest that the use of analogy in instruction can lead to better learning outcomes. Prior work on analogy and similarity offers methods that might help learners overcome their tendency to focus on superficial dissimilarities. For example,
Gick and Holyoak [37] showed that simple reminders about the similarities between two solutions could help adult college subjects make an analogy between two structurally similar, but superficially different, problems. A growing body of research suggests that there are other ways besides simply providing hints that may help children to see connections between different forms of mathematics problems, and by extension (we believe), different forms of representation of the same problem. For example, Star and Rittle-Johnson [38] demonstrated that drawing specific analogies between problems of different formats facilitates students' understanding of the underlying algebraic concepts that are common to both problems, regardless of format. Japanese teachers are much more likely to point out correspondences between different problems, whereas American teachers tend to solve the individual problems with relatively little comparison between previous and current problems [39]. Comparison, analogy, and alignment of parts of a solution can facilitate understanding of the principles that are involved in a set of problems. For example, explicitly directing children who are learning the written format for double-digit subtraction to think of the ones column as a stack of blocks could provide a valuable means for physically instantiating the concept of borrowing.

A similar idea may hold in facilitating children's understanding of the relation between concrete and abstract representations of similar problems. Pointing out correspondences between manipulatives-based procedures and comparable procedures in writing may facilitate children's understanding of both the commonalities and differences between the two forms of representation. Reminders that highlight the relational similarities between manipulative and written formats of two-digit subtraction problems might help children transfer learning between manipulatives and written representations of the same problems. Such comparisons can then form the basis of higher-order principles that are more portable and less tied to either specific context.

We do not suggest, however, that teachers can or should limit their analogies and comparison to specific correspondences between numerals and mathematical symbols and specific manipulatives. It would be a mistake, we believe, to interpret this suggestion as advocating the linkage of specific numerals or mathematical symbols to particular manipulatives [21]. That approach might lead children to focus on superficial similarities (e.g." "this block $=3$ ") rather than on more structural similarities (e.g., the process of borrowing in writing is like the process of borrowing with blocks). Thus, what we are advocating is the systematic comparison of solutions to problems involving the two forms of representation.

\section{Conclusion}

In the present work, we have demonstrated how transfer problems exist in both directions. This demonstration can be interpreted either negatively or positively. On the negative side, it means that even extensive instruction with manipulatives may not help children perform well on written tests, such as the standardized tests that they are often required to 
take in most if not all states. However, on the positive side, our results indicate that the problem is neither with manipulatives nor with written representations, but the problem is specifically with helping children to see the connection between the two. Several emerging lines of research point the way to new and more effective methods for helping children see the connection between different forms of representation.

\section{Acknowledgments}

This research was funded by the U.S. Department of Education, IES, Grant R305H050059. The authors thank Laura Mesa for her help in testing participants. The authors also thank Dedre Gentner and Nicole McNeil for their support and insights.

\section{References}

[1] G. Lakoff and R. E. Núñez, Where Mathematics Comes from: How the Embodied Mind Brings Mathematics into Being, Basic Books, New York, NY, USA, 2000.

[2] R. Gelman and C. R. Gallistel, "Language and the origin of numerical concepts," Science, vol. 306, no. 5695, pp. 441-443, 2004.

[3] D. Landy and R. L. Goldstone, "How abstract is symbolic thought?" Journal of Experimental Psychology, vol. 33, no. 4, pp. 720-733, 2007.

[4] J. S. Bruner, Toward a Theory of Instruction, Harvard University Press, Cambridge, Mass, USA, 1966.

[5] A. S. Lillard, Montessori: The Science Behind the Genius, Oxford University Press, New York, NY, USA, 2005.

[6] M. Montessori, The Advanced Montessori Method, Frederick A. Stokes, New York, NY, USA, 1917.

[7] National Council of Teachers of Mathematics, Principles and Standards for School Mathematics, National Council of Teachers of Mathematics, Reston, Va, USA, 2000.

[8] J. Piaget, Science of Education and the Psychology of the Child, Orion Press, New York, NY, USA, 1970.

[9] H. Werner and B. Kaplan, Symbol Formation: An OrganismicDevelopmental Approach to Language and the Expression of Thought, Wiley, New York, NY, USA, 1963.

[10] N. M. McNeil, D. H. Uttal, L. Jarvin, and R. J. Sternberg, "Should you show me the money? Concrete objects both hurt and help performance on mathematics problems," Learning and Instruction, vol. 19, no. 2, pp. 171-184, 2009.

[11] S.-J. Chao, J. W. Stigler, and J. A. Woodward, "The effects of physical materials on kindergartners' learning of number concepts," Cognition and Instruction, vol. 18, no. 3, pp. 285-316, 2000.

[12] C. Fuson and D. Briars, "Using a base-ten blocks learning/teaching approach for first-and second-grade place-value and multidigit addition and subtraction," Journal for Research in Mathematics Education, vol. 21, no. 3, pp. 180-206, 1990.

[13] T. Martin and D. L. Schwartz, "Physically distributed learning: adapting and reinterpreting physical environments in the development of fraction concepts," Cognitive Science, vol. 29, no. 4, pp. 587-625, 2005.

[14] L. B. Resnick and S. F. Omanson, "Learning to understand arithmetic," in Advances in Instructional Psychology, R. Glaser, Ed., vol. 3, pp. 41-95, L. Erlbaum, Hillsdale, NJ, USA, 1987.
[15] E. L. Thorndike, Animal Intelligence: Experimental Studies, Norwood Press, Norwood, Mass, USA, 1911.

[16] E. Tulving and D. M. Thomson, "Encoding specificity and retrieval processes in episodic memory," Psychological Review, vol. 80, no. 5, pp. 352-373, 1973.

[17] J. S. DeLoache, "Dual representation and young children's use of scale models," Child Development, vol. 71, no. 2, pp. 329-338, 2000.

[18] D. H. Uttal, K. V. Scudder, and J. S. DeLoache, "Manipulatives as symbols: a new perspective on the use of concrete objects to teach mathematics," Journal of Applied Developmental Psychology, vol. 18, no. 1, pp. 37-54, 1997.

[19] H. W. Stevenson, S.-Y. Lee, and J. W. Stigler, "Mathematics achievement of Chinese, Japanese, and American children," Science, vol. 231, no. 4739, pp. 693-699, 1986.

[20] J. Hiebert and D. Wearne, "Procedures over concepts: the acquisition of decimal number knowledge," in Conceptual and Procedural Knowledge: the Case of Mathematics, J. Hiebert, Ed., pp. 199-223, Lawrence Erlbaum, Hillsdale, NJ, USA, 1986.

[21] M. Hughes, Children and Number: Difficulties in Learning Mathematics, Basil Blackwell, Oxford, UK, 1986.

[22] D. H. Uttal, L. L. Liu, and J. S. Deloache, "Concreteness and symbolic development," in Child Psychology: A Handbook of Contemporary Issues, L. Balter and C. S. Tamis-LeMonda, Eds., pp. 167-184, Psychology Press, Philadelphia, Pa, USA, 2nd edition, 2006.

[23] J. S. DeLoache, "Rapid change in the symbolic functioning of very young children," Science, vol. 238, no. 4833, pp. 1556-1557, 1987.

[24] J. S. DeLoache, "Young children's understanding of the correspondence between a scale model and a larger space," Cognitive Development, vol. 4, no. 2, pp. 121-139, 1989.

[25] J. S. Deloache, "Early symbol understanding and use," in The Psychology of Learning and Motivation: Advances in Research and Theory, D. L. Medin, Ed., vol. 33, pp. 65-114, Academic Press, San Diego, Calif, USA, 1995.

[26] A. S. Dick, W. F. Overton, and S. L. Kovacs, “The development of symbolic coordination: representation of imagined objects, executive function, and theory of mind," Journal of Cognition and Development, vol. 6, no. 1, pp. 133-161, 2005.

[27] J. A. Kaminski and V. M. Sloutsky, "Extraneous perceptual information interferes with children's acquisition of mathematical knowledge," Journal of Educational Psychology, vol. 105, no. 2, p. 351, 2013.

[28] J. A. Kaminski, V. M. Sloutsky, and A. F. Heckler, "The advantage of abstract examples in learning math," Science, vol. 320, no. 5875, pp. 454-455, 2008.

[29] D. Corbiere, Digi-Block Companion to Everyday Mathematics: Grade 1, Digi-Blocks, Watertown, Mass, USA, 2003.

[30] P. Noble, Digi-Block Companion to Everyday Mathematics: Grade 2, Digi-Blocks, Watertown, Mass, USA, 2003.

[31] J. Davidson, Using the Cuisenaire Rods: A Photo/Text Guide for Teachers, Cuisenaire Company of America, New Rochelle, NY, USA, 1983.

[32] M. Bell, J. Bell, J. Bretzlauf et al., Everyday Mathematics: Second Grade Teacher's lesson Guide, vol. 1, Everyday Learning Corporation, Chicago, Ill, USA, 2001.

[33] P. Dugard and J. Todman, "Analysis of pretest posttest control group designs in educational research," Educational Psychology, vol. 15, no. 2, p. 181, 1995. 
[34] E. J. Sowell, "Effects of manipulative materials in mathematics instruction," Journal for Research in Mathematics Education, vol. 20, pp. 498-505, 1989.

[35] D. L. Ball, "Magical hopes: manipulatives and the reform of math education," American Educator, vol. 16, no. 2, pp. 14-18, 1992.

[36] D. H. Clements and S. McMillen, "Rethinking, "concrete" manipulatives," Teaching Children Mathematics, vol. 2, no. 5, pp. 270-279, 1996.

[37] M. L. Gick and K. J. Holyoak, "Analogical problem solving," Cognitive Psychology, vol. 12, no. 3, pp. 306-355, 1980.

[38] J. R. Star and B. Rittle-Johnson, "Flexibility in problem solving: the case of equation solving," Learning and Instruction, vol. 18, no. 6, pp. 565-579, 2008.

[39] L. E. Richland, O. Zur, and K. J. Holyoak, "Cognitive supports for analogies in the mathematics classroom," Science, vol. 316, no. 5828, pp. 1128-1129, 2007. 

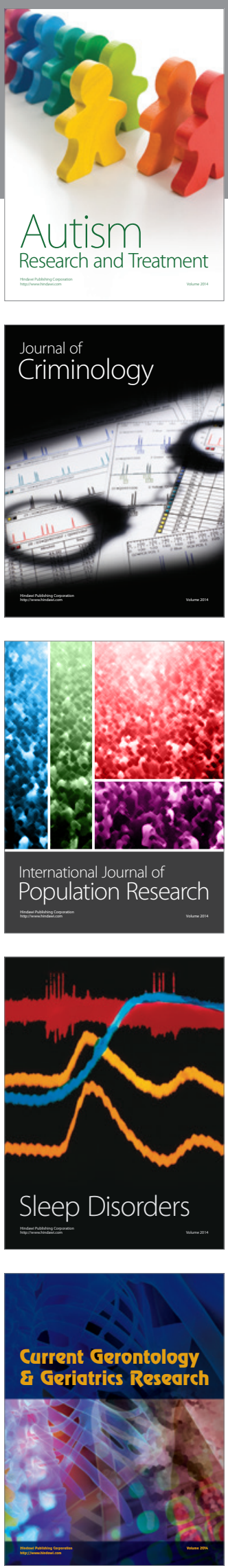
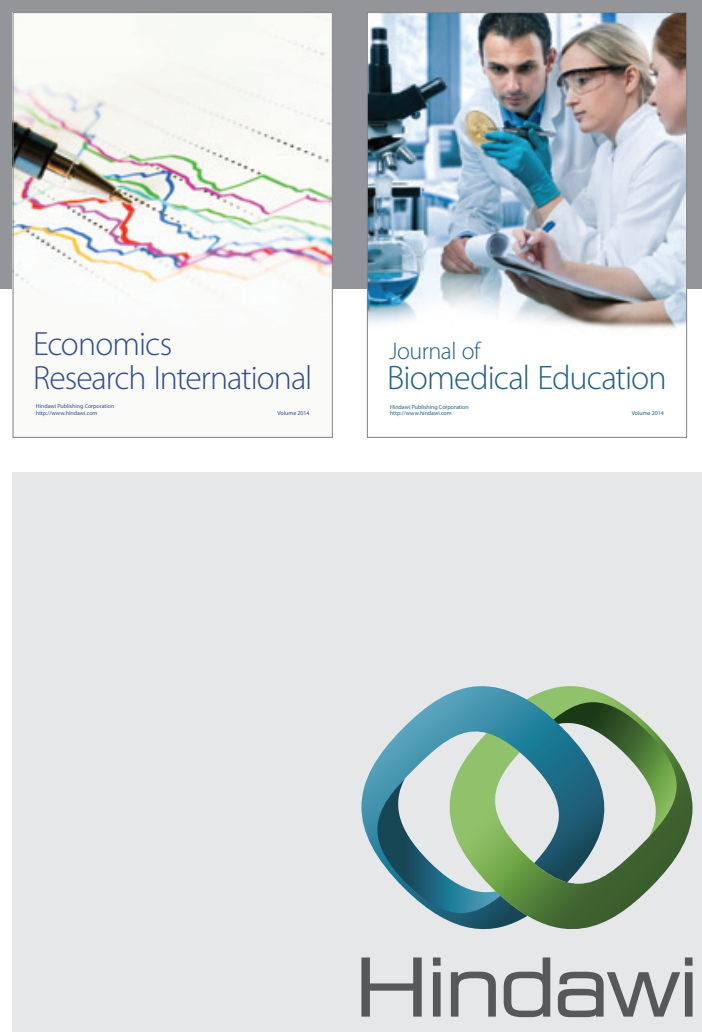

Submit your manuscripts at

http://www.hindawi.com
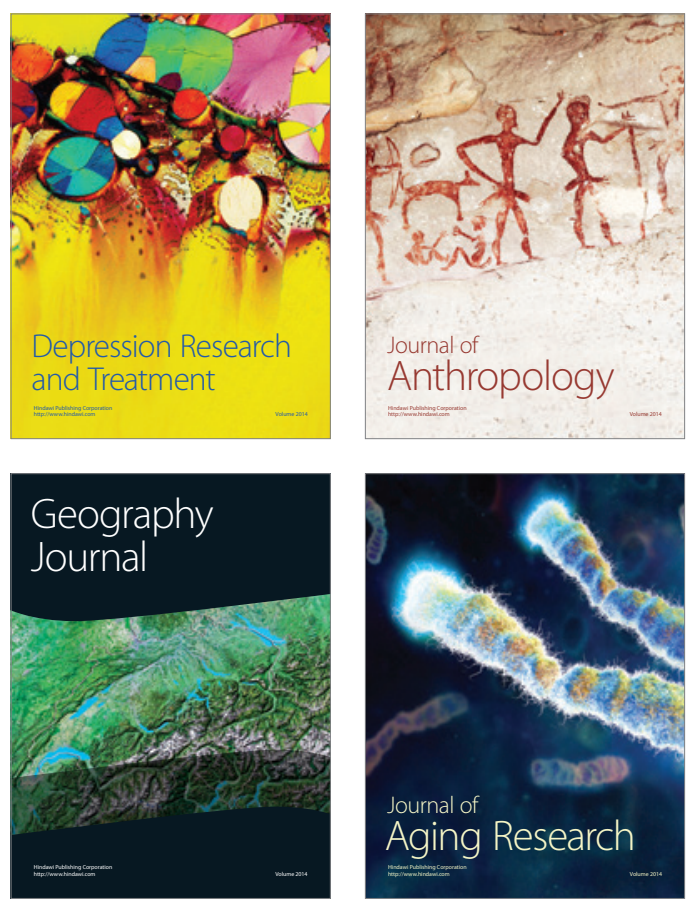
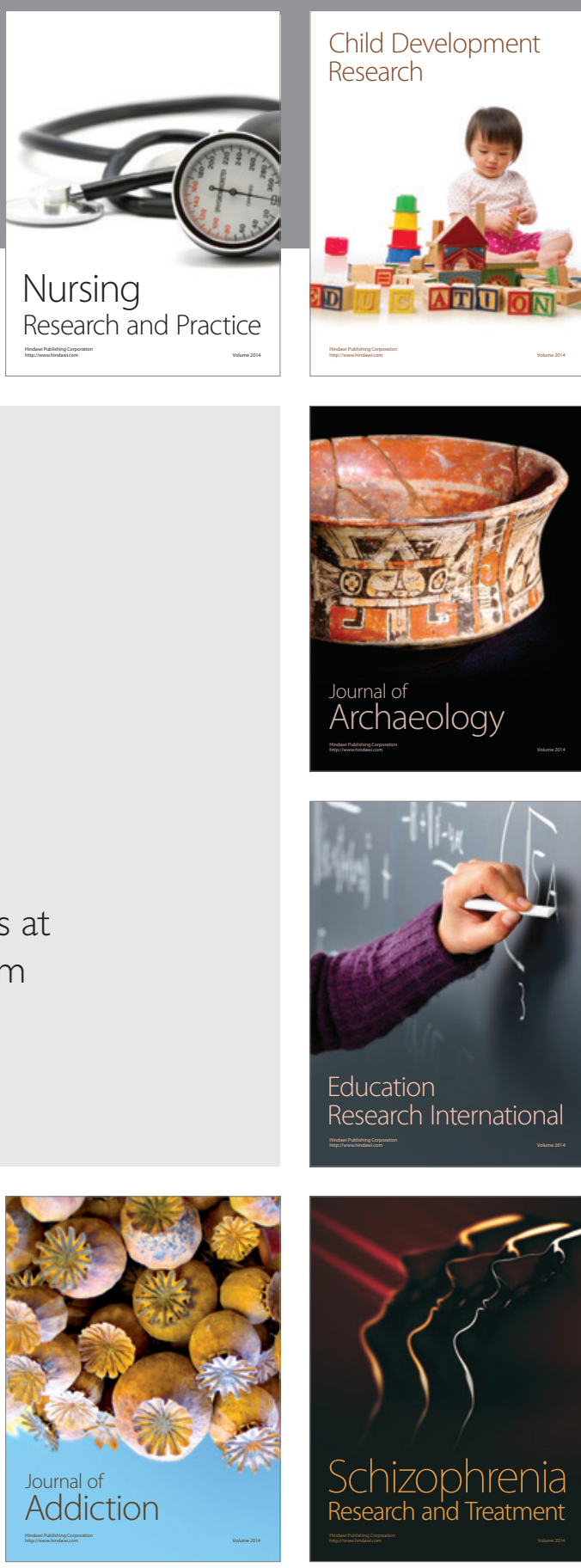

(D)
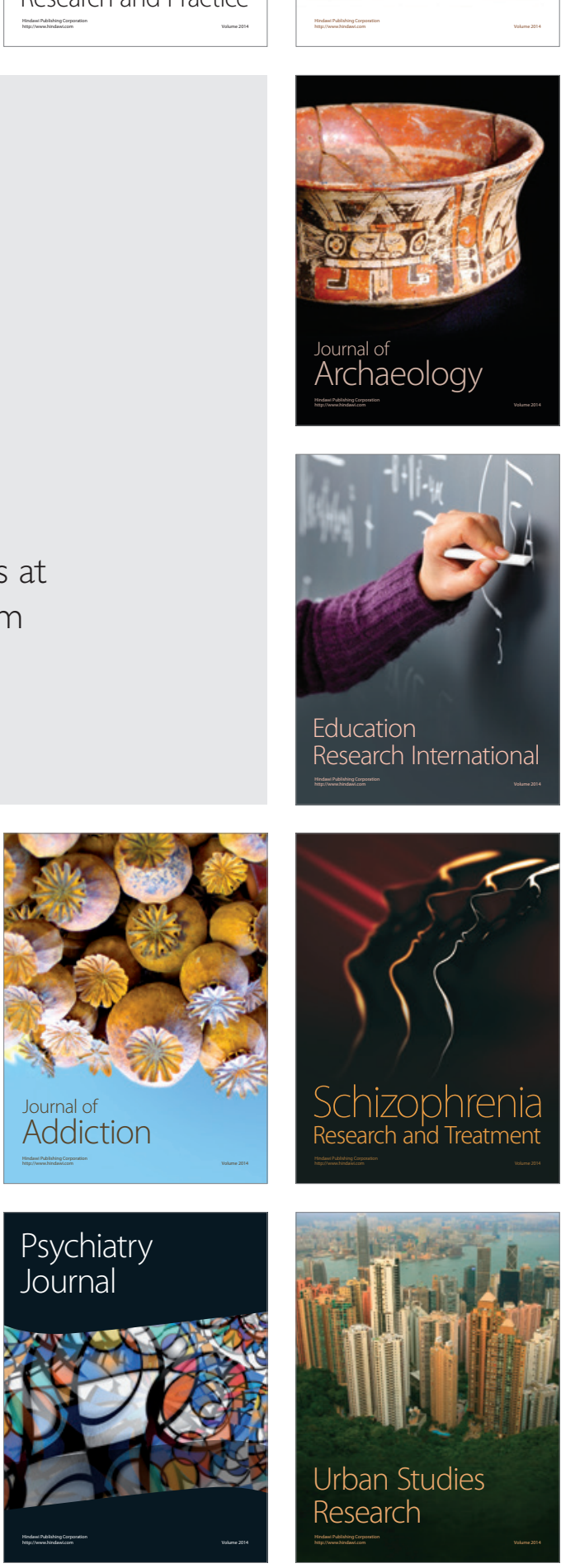